\title{
Spin-orbit torques originating from the bulk and interface in Pt-based structures
}

\author{
Hiroki Hayashi, ${ }^{1}$ Akira Musha, ${ }^{1}$ Hiroto Sakimura,,${ }^{1,2}$ and Kazuya Ando ${ }^{1,3,4, *}$ \\ ${ }^{1}$ Department of Applied Physics and Physico-Informatics, Keio University, Yokohama 223-8522, Japan \\ ${ }^{2}$ School of Materials and Chemical Technology, Tokyo Institute of Technology, Tokyo 152-8522, Japan \\ ${ }^{3}$ Keio Institute of Pure and Applied Science, Keio University, Yokohama 223-8522, Japan \\ ${ }^{4}$ Center for Spintronics Research Network, Keio University, Yokohama 223-8522, Japan
}

(Received 6 April 2020; revised 18 December 2020; accepted 21 December 2020; published 13 January 2021)

\begin{abstract}
We investigate spin-orbit torques in prototypical Pt-based spintronic devices. We find that, in $\mathrm{Pt} / \mathrm{Ni}$ and $\mathrm{Pt} / \mathrm{Fe}$ bilayers, the dampinglike torque efficiency depends on the thickness of the Pt layer. We also find that the dampinglike torque efficiency is almost identical in the $\mathrm{Pt} / \mathrm{Ni}$ and $\mathrm{Pt} / \mathrm{Fe}$ bilayers despite the stronger spin memory loss at the $\mathrm{Pt} / \mathrm{Fe}$ interface. These results suggest that although the dominant source of the dampinglike torque is the bulk spin Hall effect in the Pt layer, a sizable dampinglike torque is generated by the interface in the $\mathrm{Pt} / \mathrm{Fe}$ bilayer due to the stronger interfacial spin-orbit coupling. In contrast to the dampinglike torque, whose magnitude and sign are almost identical in the $\mathrm{Pt} / \mathrm{Ni}$ and $\mathrm{Pt} / \mathrm{Fe}$ bilayers, the fieldlike torque strongly depends on the choice of the ferromagnetic layer. The sign of the fieldlike torque originating from the bulk spin Hall effect in the Pt layer is opposite between the $\mathrm{Pt} / \mathrm{Ni}$ and $\mathrm{Pt} / \mathrm{Fe}$ bilayers, which can be attributed to the opposite sign of the imaginary part of the spin-mixing conductance. These results demonstrate that the spin-orbit torques are quite sensitive to the electronic structure of the ferromagnet layer.
\end{abstract}

DOI: 10.1103/PhysRevResearch.3.013042

\section{INTRODUCTION}

Current-induced spin-orbit torques provide a promising strategy for the electrical manipulation of magnetization in metals, semiconductors, and insulators [1-16]. The efficient manipulation of magnetization through the spin-orbit torques offers a path for ultralow power, fast nonvolatile magnetic memory, and logic technologies [17]. The spin-orbit torques arise from the transfer of orbital angular momentum from the lattice to the spin system, which results from spin-orbit coupling in the bulk and at the interface of heavymetal/ferromagnet (HM/FM) bilayers [1].

The bulk spin-orbit coupling in the HM causes carriers with opposite spins to scatter in opposite directions. The spindependent scattering generates a spin current from a charge current, which is known as the spin Hall effect [18-28]. In the $\mathrm{HM} / \mathrm{FM}$ bilayer, the angular momentum carried by the spin Hall current is transferred to the magnetization through the spin-transfer mechanism [1]. This angular momentum transfer induces a torque on the magnetization, which is expressed as $\boldsymbol{\tau}_{\mathrm{DL}}=\tau_{\mathrm{DL}} \mathbf{m} \times(\boldsymbol{\sigma} \times \mathbf{m})$, where $\mathbf{m}$ is the magnetization unit vector, $\sigma$ is the unit vector along the spin-polarization direction of the spin current, and $\tau_{\mathrm{DL}}$ is the magnitude of the torque. The torque of this form is referred to as a dampinglike torque. The transfer of the angular momentum is generally imperfect

\footnotetext{
*ando@appi.keio.ac.jp

Published by the American Physical Society under the terms of the Creative Commons Attribution 4.0 International license. Further distribution of this work must maintain attribution to the author(s) and the published article's title, journal citation, and DOI.
}

at the interface partly because of a small component of the spins that rotate when they reflect from the interface [29]. The spin rotation at the interface gives rise to a torque in the form of $\boldsymbol{\tau}_{\mathrm{FL}}=\tau_{\mathrm{FL}} \boldsymbol{\sigma} \times \mathbf{m}$, which is referred to as a fieldlike torque.

The interfacial spin-orbit coupling in the HM/FM bilayer also generates the dampinglike and fieldlike torques. At the interface with broken inversion symmetry, the spin-orbit coupling lifts the electron-spin degeneracy and the spin angular momentum is locked on the linear momentum [30,31]. Because of the spin-momentum locking, a charge current flowing parallel to the interface generates a nonzero spin accumulation [32,33]. This process, called the Rashba-Edelstein or inverse spin galvanic effect, exerts a torque on the magnetization through the exchange coupling at the HM/FM interface $[2,34,35]$. Since the interfacial Rashba spin-orbit effective field induces the rotation of the spin accumulation, both fieldlike and dampinglike torques can be generated by the current-induced spin accumulation and exchange coupling [5,36-40]. Although in this scenario carriers are assumed to be confined to the two-dimensional interface, in the HM/FM bilayer carriers are not confined but can be scattered across the interface. In this situation, the interfacial spin-orbit coupling can also generate an interfacial spin current that flows away from the FM/HM interface through interfacial spinorbit scattering. The interfacial spin-orbit scattering generates both dampinglike and fieldlike torques [41].

Understanding the physics behind the generation of the spin-orbit torques is essential for the development of spinorbitronic devices, as well as the fundamental understanding of spin-dependent transport in condensed matter. A wide range of experiments has demonstrated that the spin-orbit torques can be manipulated by materials and interface engineering in Pt-based structures [42-47], where the spin-orbit 
torques are generally attributed to the strong spin-orbit coupling of $\mathrm{Pt}$, the most widely studied source of spin currents. However, despite this progress, the origin of the spin-orbit torques is still unclear and controversial even in the prototypical spin-orbitronic device. A typical example is the fieldlike torque in Pt/Ni-Fe-alloy bilayers [43,44,48,49]. The reported values vary significantly for the same system and even the sign, as well as the magnitude, is inconsistent in the literature, implying an important role of the spin-orbit coupling and electronic structure of the FM layer in the generation of the spin-orbit torques.

In this paper we show that the origin of the spin-orbit torques in the standard Pt/FM bilayer strongly depends on the choice of the FM. We find that the dampinglike torque efficiency depends on the thickness of the Pt layer in $\mathrm{Pt} / \mathrm{Ni}$ and $\mathrm{Pt} / \mathrm{Fe}$ bilayers. We also find that the dampinglike torque efficiency is almost identical in the $\mathrm{Pt} / \mathrm{Ni}$ and $\mathrm{Pt} / \mathrm{Fe}$ bilayers despite the stronger spin memory loss at the $\mathrm{Pt} / \mathrm{Fe}$ interface. These results suggest that although the dominant source of the dampinglike torque is the bulk spin Hall effect in the Pt layer, a sizable dampinglike torque is generated by the interface in the $\mathrm{Pt} / \mathrm{Fe}$ bilayer due to the stronger interfacial spin-orbit coupling. We also find that the sign of the fieldlike torque originating from the bulk spin Hall effect in the Pt layer is opposite between the $\mathrm{Pt} / \mathrm{Ni}$ and $\mathrm{Pt} / \mathrm{Fe}$ bilayers, which can be attributed to the opposite sign of the imaginary part of the spin-mixing conductance. Although the strong spin-orbit coupling of the Pt layer is expected to play an essential role in the prototypical Pt-based structure, these results demonstrate that the spin-orbit torques are quite sensitive to the electronic structure of the FM layer. These results provide a crucial piece of information for a fundamental understanding of the spin-orbit torques.

\section{EXPERIMENTAL METHOD}

We investigated the spin-orbit torques in $\mathrm{Pt} / \mathrm{Ni}$ and $\mathrm{Pt} / \mathrm{Fe}$ bilayers using spin-torque ferromagnetic resonance (STFMR). The sample structure was $\mathrm{Ti}(2) / \mathrm{Pt}\left(d_{\mathrm{Pt}}\right) / \mathrm{FM}\left(d_{\mathrm{FM}}\right) / \mathrm{SiO}_{2}(5)$, where the numbers in parentheses represent the thickness in units of nanometers [see Fig. 1(a)]. In addition, $d_{\mathrm{Pt}}$ and $d_{\mathrm{FM}}$ are the thicknesses of the $\mathrm{Pt}$ and FM layers, respectively. The films were deposited on thermally oxidized $\mathrm{Si} / \mathrm{SiO}_{2}(100)$ substrates using RF-magnetron sputtering, where the base pressure was around $1 \times 10^{-5} \mathrm{~Pa}$. The 2-nm-thick Ti layer was sputtered on the substrate and then the Pt layer was sputtered on the adhesion Ti layer, where the deposition rate of $\mathrm{Ti}(\mathrm{Pt})$ was 0.01 $(0.03) \mathrm{nm} / \mathrm{s}$. On the Pt layer, the FM layer $(\mathrm{FM}=\mathrm{Ni}$ and $\mathrm{Fe})$ was sputtered, where the deposition rate of $\mathrm{Ni}(\mathrm{Fe})$ was 0.04 $(0.02) \mathrm{nm} / \mathrm{s}$. To avoid the natural oxidation of the FM layer, the 5-nm-thick $\mathrm{SiO}_{2}$ was sputtered on the FM layer, where the deposition rate was $0.01 \mathrm{~nm} / \mathrm{s}$. All the sputtering processes were conducted in the $5 \mathrm{~N}$-purity argon atmosphere of $0.4 \mathrm{~Pa}$ at room temperature.

To measure the STFMR, the Pt/FM films were patterned into rectangular shapes $\left(10 \times 150 \mu \mathrm{m}^{2}\right)$ with $\mathrm{Ti}(2) / \mathrm{Pt}(60)$ electrodes using the conventional photolithography and liftoff technique. For the STFMR measurement, we applied an RF current with the frequency $f$ along the longitudinal

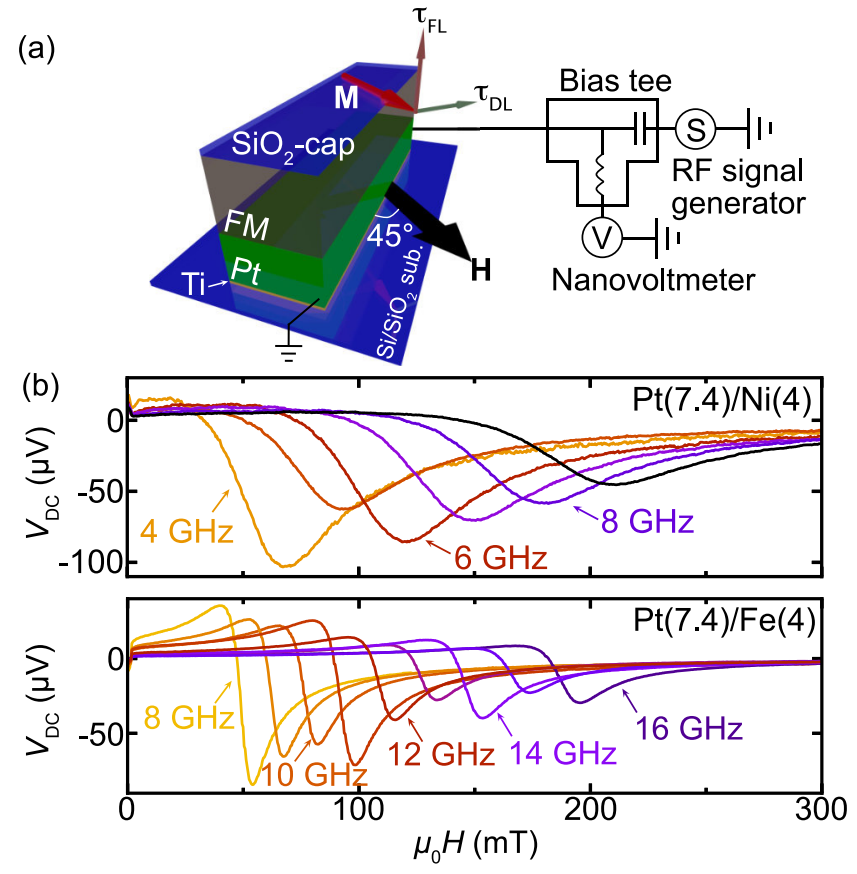

FIG. 1. (a) Schematic illustration of the experimental setup of the STFMR measurement for the Pt/FM (FM = Ni and Fe) bilayers. (b) Magnetic field $H$ dependence of the DC voltage $V_{\mathrm{DC}}$ for the $\mathrm{Pt}(7.4) / \mathrm{Ni}(4)$ (upper) and $\mathrm{Pt}(7.4) / \mathrm{Fe}(4)$ (lower) bilayers measured with an RF power of $24.7 \mathrm{dBm}$. The RF frequency was varied from $f=4$ to $9 \mathrm{GHz}$ for the $\mathrm{Pt} / \mathrm{Ni}$ film and from $f=8$ to $16 \mathrm{GHz}$ for the $\mathrm{Pt} / \mathrm{Fe}$ film. The measurement of the STFMR for the Pt/Ni film at higher $f$ requires higher $H$, which limits the $f$ range in the present study.

direction of the Pt/FM bilayer and an external magnetic field $\mathbf{H}$ at an angle of $45^{\circ}$ from the longitudinal direction, as shown in Fig. 1(a). The RF current generates out-of-plane and in-plane torques due to the spin-orbit torques and an Oersted field, which drive the magnetization precession under the FMR conduction. The precession of the magnetization leads to the oscillation of the resistance of the device through the anisotropic magnetoresistance (AMR) of the FM layer. In the bilayer, the mixing of the RF current and oscillating resistance induces DC voltage $V_{\mathrm{DC}}$. We measured $V_{\mathrm{DC}}$ for the $\mathrm{Pt} / \mathrm{FM}$ bilayers using a bias tee and a nanovoltmeter at room temperature, as shown in Fig. 1(a). Here the phase of the RF current is almost constant in the STFMR devices because the wavelength of the RF current with a frequency of 4-16 GHz is much larger than the length of the devices, $150 \mu \mathrm{m}$. In fact, a previous study demonstrated that the phase of the magnetization precession driven by an RF current is almost constant in a STFMR device with a width of $100 \mu \mathrm{m}$ and a length of $400 \mu \mathrm{m}$ [50], supporting that the current nonuniformity is negligible in the present study.

The STFMR voltage $V_{\mathrm{DC}}$ can be decomposed into two components [51]

$$
\begin{aligned}
V_{\mathrm{DC}}= & A \frac{W\left(\mu_{0} H-\mu_{0} H_{\mathrm{FMR}}\right)}{\left(\mu_{0} H-\mu_{0} H_{\mathrm{FMR}}\right)^{2}+W^{2}} \\
& +S \frac{W^{2}}{\left(\mu_{0} H-\mu_{0} H_{\mathrm{FMR}}\right)^{2}+W^{2}},
\end{aligned}
$$


where $W$ is the spectral width and $H_{\mathrm{FMR}}$ is the FMR field. The symmetric $S$ and antisymmetric $A$ components are produced by the out-of-plane and in-plane effective fields, respectively. Here the out-of-plane effective field is the dampinglike effective field $H_{\mathrm{DL}}$ and the in-plane effective field is the sum of the Oersted field $H_{\mathrm{Oe}}$ due to the current flow in the Pt layer and the fieldlike effective field $H_{\mathrm{FL}}$. The dampinglike and fieldlike torque efficiencies per applied electric field $E$, defined as

$$
\xi_{\mathrm{DL}(\mathrm{FL})}^{E}=\left(\frac{2 e}{\hbar}\right) \frac{\mu_{0} M_{\mathrm{s}} d_{\mathrm{FM}} H_{\mathrm{DL}(\mathrm{FL})}}{E},
$$

can be determined by measuring the STFMR for devices with different $d_{\mathrm{FM}}$ using [52]

$$
\frac{1}{\xi_{\mathrm{FMR}}}=\frac{1}{\xi_{\mathrm{DL}}^{E}}\left(\frac{1}{\rho_{\mathrm{Pt}}}+\frac{\hbar}{e} \frac{\xi_{\mathrm{FL}}^{E}}{\mu_{0} M_{\mathrm{s}} d_{\mathrm{FM}} d_{\mathrm{Pt}}}\right),
$$

where $e$ is the elementary charge, $\hbar$ is the Dirac constant, $\rho_{\mathrm{Pt}}$ is the electric resistivity of the Pt layer, $M_{s}$ is the saturation magnetization, and

$$
\xi_{\mathrm{FMR}}=\frac{S}{A} \frac{e \mu_{0} M_{s} d_{\mathrm{FM}} d_{\mathrm{Pt}}}{\hbar} \sqrt{1+\frac{M_{\mathrm{eff}}}{H_{\mathrm{FMR}}}}
$$

is the FMR spin-torque generation efficiency, where $M_{\text {eff }}$ is the effective demagnetization field. The resistivity $\rho_{\mathrm{Pt}}$ of the $\mathrm{Pt}$ layer in the Pt/FM bilayers was determined by the standard four-probe method for single Pt films, sputtered on the Ti layer, where the sheet resistance of the Ti layer was orders of magnitude larger than that of the Pt layer. The resistivity of the Pt layer was $\rho_{\mathrm{Pt}}=65.6 \mu \Omega \mathrm{cm}$ for $d_{\mathrm{Pt}}=1.5 \mathrm{~nm}$ and $\rho_{\mathrm{Pt}}=23.0 \mu \Omega \mathrm{cm}$ for $d_{\mathrm{Pt}}=7.4 \mathrm{~nm}$. The increase of the resistivity induced by decreasing the thickness $d_{\mathrm{Pt}}$ can be attributed to the surface scattering, rather than a possible discontinuity of the Pt layer. In fact, the difference in the resistivity between the Pt layers with $d_{\mathrm{Pt}}=1.5$ and $7.4 \mathrm{~nm}$ is in good agreement with that reported previously, where the drift-diffusion model is consistent with the experimental result for $\mathrm{Pt} / \mathrm{Co}$ bilayers with $1.2 \mathrm{~nm}<d_{\mathrm{Pt}}<15 \mathrm{~nm}$ [53].

\section{RESULTS AND DISCUSSION}

\section{A. Spin-torque ferromagnetic resonance}

In Fig. 1(b) we show the magnetic field $H$ dependence of the DC voltage $V_{\mathrm{DC}}$ for the $\mathrm{Pt}(7.4) / \mathrm{Ni}(4)$ and $\mathrm{Pt}(7.4) / \mathrm{Fe}(4)$ bilayers. We first characterize the DC voltage $V_{\mathrm{DC}}$ for the $\mathrm{Pt} / \mathrm{FM}$ devices by measuring RF current power $P$, magnetic field angle $\theta$, and RF current frequency $f$ dependence of the STFMR.

Figures 2(a) and 2(b) show the RF power $P$ dependence of the STFMR signal for the $\mathrm{Pt}(7.4) / \mathrm{Fe}(5)$ and $\mathrm{Pt}(7.4) / \mathrm{Ni}(4)$ bilayers, where the symmetric $S$ and antisymmetric $A$ components of $V_{\mathrm{DC}}$ were obtained by fitting the measured $V_{\mathrm{DC}}$ using Eq. (1). This result shows that both symmetric $S$ and antisymmetric $A$ components are proportional to $P$, indicating that the STFMR is in the linear response regime. We also note that the FMR field $H_{\mathrm{FMR}}$ is independent of the applied microwave power $P$ as shown in Figs. 2(b) and 2(c). The negligible change of $H_{\mathrm{FMR}}$ shows that the sample heating due to the microwave application and absorption barely affects the magnetic properties of the STFMR devices. (a)

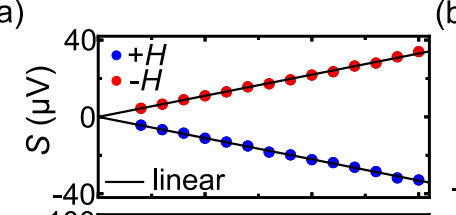

(b)

(c)
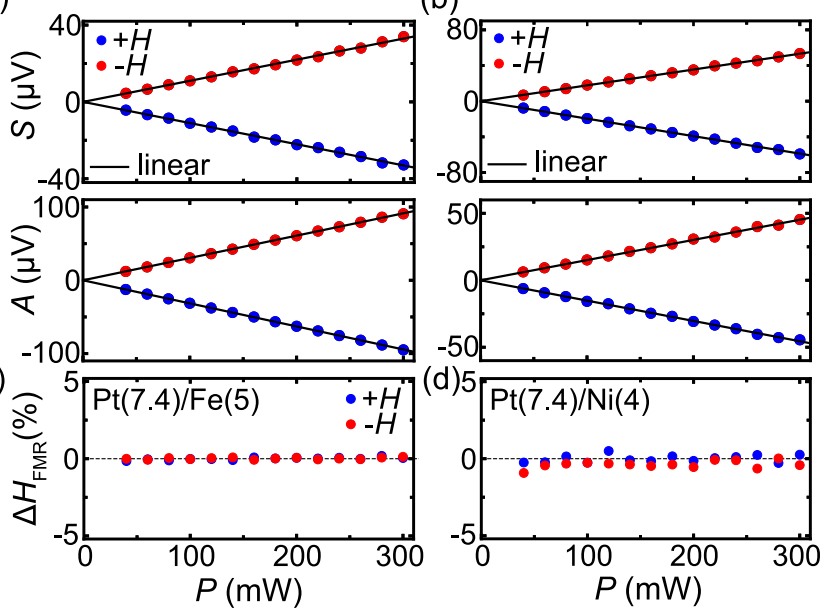

FIG. 2. The RF power $P$ dependence of the symmetric $S$ (upper) and antisymmetric $A$ (lower) components of the DC voltage $V_{\mathrm{DC}}$ for (a) the $\mathrm{Pt}(7.4) / \mathrm{Fe}(5)$ bilayer at an $\mathrm{RF}$ of $f=10 \mathrm{GHz}$ and (b) the $\mathrm{Pt}(7.4) / \mathrm{Ni}(4)$ bilayer at $f=7 \mathrm{GHz}$. The solid lines are the linear fitting result. Also shown is the RF power $P$ dependence of the change ratio of the FMR field $\Delta H_{\mathrm{FMR}}=\left[H_{\mathrm{FMR}}(P)-\bar{H}_{\mathrm{FMR}}\right] / \bar{H}_{\mathrm{FMR}}$ for the (c) $\mathrm{Pt}(7.4) / \mathrm{Ni}(4)$ and (d) $\mathrm{Pt}(7.4) \mathrm{Fe}(5)$ bilayers, where $\bar{H}_{\mathrm{FMR}}$ represents the averaged value of $H_{\mathrm{FMR}}(P)$.

In Figs. 3(a) and 3(b) we show the magnetic field angle $\theta$ dependence of the symmetric $S$ and antisymmetric $A$ voltage, where the in-plane external magnetic field $\mathbf{H}$ was applied at the angle of $\theta$ from the longitudinal direction of the $\mathrm{Pt} / \mathrm{Fe}$ (a)
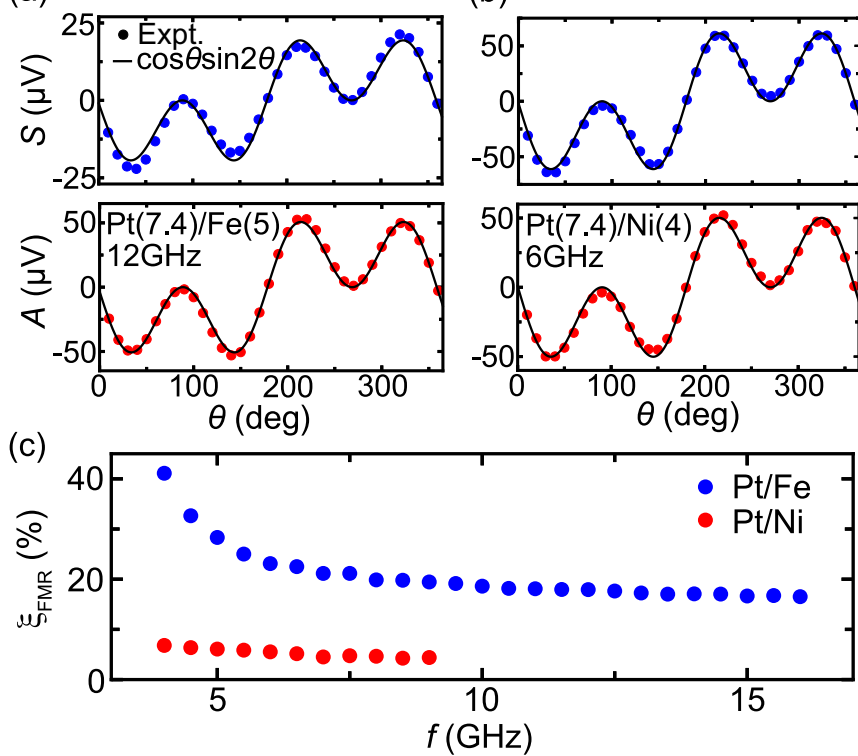

(b)
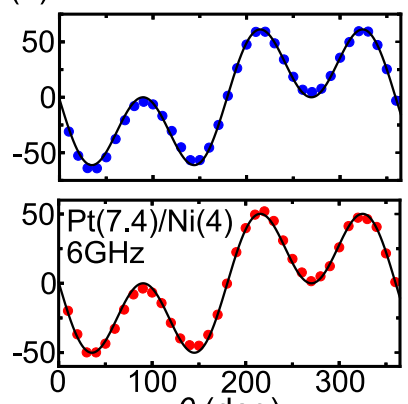

FIG. 3. The $S$ and $A$ components of the STFMR spectra as a function of the in-plane magnetic field angle $\theta$ for (a) the $\mathrm{Pt}(7.4) / \mathrm{Fe}(5)$ film at $f=12 \mathrm{GHz}$ and (b) the $\mathrm{Pt}(7.4) \mathrm{Ni}(4)$ film at $f=6 \mathrm{GHz}$. Here the in-plane external magnetic field $\mathbf{H}$ was applied at an angle of $\theta$ from the longitudinal direction. (c) The RF $f$ dependence of the FMR spin-torque generation efficiency $\xi_{\text {FMR }}$ from $f=4$ to $16 \mathrm{GHz}$ for the $\mathrm{Pt}(7.4) / \mathrm{Fe}(5)$ and from $f=4$ to $9 \mathrm{GHz}$ for the $\mathrm{Pt}(7.4) / \mathrm{Ni}(5)$ bilayers. 
(a)

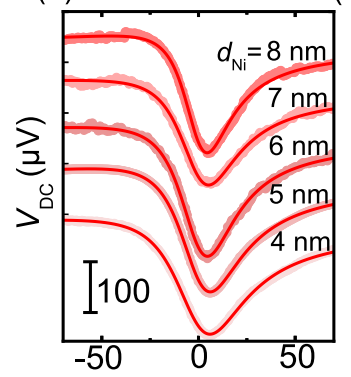

(b)

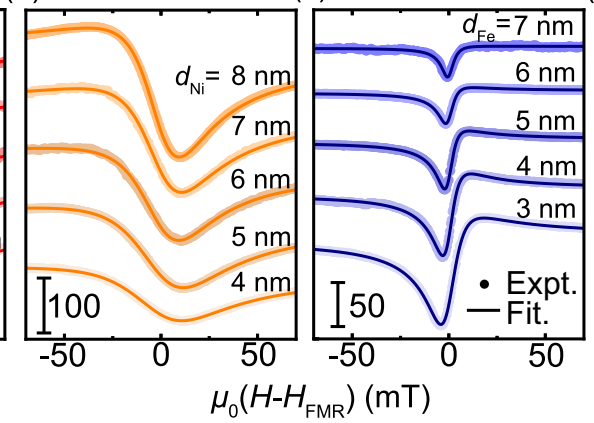

(d)

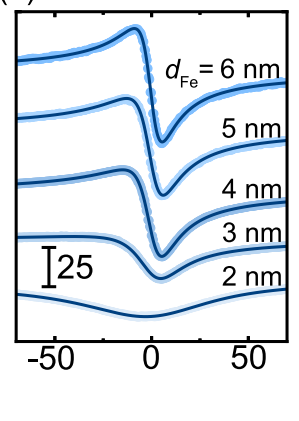

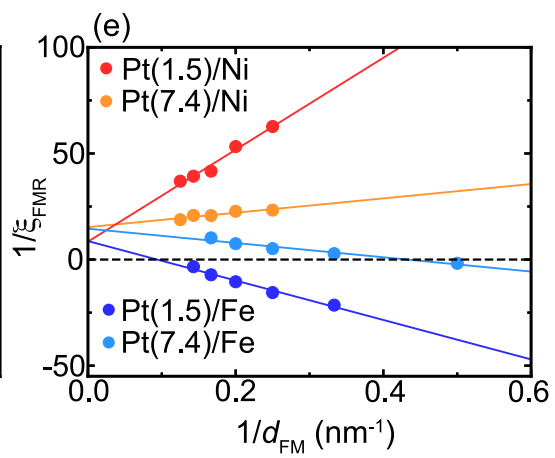

FIG. 4. Magnetic field $H$ dependence of the DC voltage $V_{\mathrm{DC}}$ for (a) $\mathrm{Pt}(1.5) / \mathrm{Ni}\left(d_{\mathrm{Ni}}\right)$ at $f=6 \mathrm{GHz}$, (b) $\mathrm{Pt}(7.4) / \mathrm{Ni}\left(d_{\mathrm{Ni}}\right)$ at $f=6 \mathrm{GHz}$, (c) $\mathrm{Pt}$ $(1.5) / \mathrm{Fe}\left(d_{\mathrm{Fe}}\right)$ at $f=12 \mathrm{GHz}$, and $(\mathrm{d}) \operatorname{Pt}(7.4) / \mathrm{Fe}\left(d_{\mathrm{Fe}}\right)$ at $f=12 \mathrm{GHz}$. The closed circles are the experimental data and the solid curves are the fitting result using Eq. (1). (e) The $1 / d_{\mathrm{FM}}$ dependence of $1 / \xi_{\mathrm{FMR}}$ for the $\mathrm{Pt} / \mathrm{Ni}$ and $\mathrm{Pt} / \mathrm{Fe}$ bilayers, where $d_{\mathrm{FM}}$ is the thickness of the FM layer and $\xi_{\mathrm{FMR}}$ is the FMR spin-torque efficiency at $f=9 \mathrm{GHz}$ for the $\mathrm{Pt} / \mathrm{Ni}$ bilayer and $f=16 \mathrm{GHz}$ for the $\mathrm{Pt} / \mathrm{Fe}$ bilayer. The closed circles are the experimental data and the solid lines are the fitting result using Eq. (3).

and $\mathrm{Pt} / \mathrm{Ni}$ devices. In both films, $S$ and $A$ are proportional to $\cos \theta \sin 2 \theta$, consistent with previous results [54]. This result indicates that the contribution from an out-of-plane Oersted field is negligible in the observed voltage, supporting that the RF current flow is uniform in the STFMR devices.

The STFMR spectra at various frequencies $f$ are shown in Fig. 1(b). This result shows that the FMR field $H_{\mathrm{FMR}}$ changes systematically by changing $f$, which is consistent with the Kittel formula $2 \pi f=\gamma \sqrt{\mu_{0} H_{\mathrm{FMR}}\left(\mu_{0} H_{\mathrm{FMR}}+\mu_{0} M_{\mathrm{eff}}\right)}$, where $\gamma$ is the gyromagnetic ratio. From the measured $V_{\mathrm{DC}}$, we calculated the FMR spin-torque generation efficiency $\xi_{\mathrm{FMR}}$ at each $f$ using Eq. (4). The $f$ dependence of $\xi_{\text {FMR }}$ for the $\mathrm{Pt} / \mathrm{FM}$ bilayers is shown in Fig. 3(c). Figure 3(c) shows that $\xi_{\text {FMR }}$ is almost independent of $f$ in the $\mathrm{Pt} / \mathrm{Ni}$ bilayer, while $\xi_{\mathrm{FMR}}$ is independent of $f$ only at higher frequency in the $\mathrm{Pt} / \mathrm{Fe}$ bilayer. Here the STFMR predicts that $\xi_{\mathrm{FMR}}$ is independent of $f$ [51]. The result for the $\mathrm{Pt} / \mathrm{Ni}$ bilayer is consistent with this prediction, showing that the measured $V_{\mathrm{DC}}$ is dominated by the STFMR for the Pt/Ni bilayer. The $f$ dependence of $\xi_{\text {FMR }}$ for the $\mathrm{Pt} / \mathrm{Fe}$ bilayer at the higher frequency is also consistent with the prediction of the STFMR. In contrast, $\xi_{\text {FMR }}$ at the lower frequency for the $\mathrm{Pt} / \mathrm{Fe}$ bilayer cannot be entirely attributed to the STFMR. In the Pt/Fe bilayer, the increase of $\xi_{\mathrm{FMR}}$ at the lower frequency can be attributed to additional contributions, including thermal effects and the inverse spin Hall effect induced by spin pumping; because of the smaller AMR of the Pt/Fe film, compared to that of the $\mathrm{Pt} / \mathrm{Ni}$ film, these additional effects can contribute to the observed $S$ in the $\mathrm{Pt} / \mathrm{Fe}$ film. Since these contributions decrease with increasing $f$ [55], these additional contributions become negligible and the STFMR dominates the measured $\xi_{\mathrm{FMR}}$ at higher frequency $f$, resulting in the $f$-independent $\xi_{\text {FMR }}$ in the $\mathrm{Pt} / \mathrm{Fe}$ film. The negligible contribution from the spin pumping and thermal effects at the higher frequency is also supported by the $1 / d_{\mathrm{FM}}$ dependence of $1 / \xi_{\mathrm{FMR}}$ as described below. In the following, we discuss the STFMR measured at the RF $f$ where $\xi_{\mathrm{FMR}}$ is almost independent of $f$ to neglect the contribution from the spin pumping and thermal effects.

In the following discussion, we neglect the spin-orbit torques arising from the $\mathrm{FM} / \mathrm{SiO}_{2}$ interface since, in the $\mathrm{Pt} / \mathrm{FM} /$ oxide structure, the contribution from the $\mathrm{FM} / \mathrm{SiO}_{2}$ interface to the spin-orbit torques is negligible compared to that from the Pt bulk and/or the Pt/FM interface [56].

\section{B. Spin-torque efficiencies}

In Figs. 4(a) and 4(b) we show the $V_{\mathrm{DC}}$ spectra at $f=$ $6 \mathrm{GHz}$ for the $\operatorname{Pt}(1.5) / \mathrm{Ni}\left(d_{\mathrm{FM}}\right)$ and $\operatorname{Pt}(7.4) / \mathrm{Ni}\left(d_{\mathrm{FM}}\right)$ bilayers with various $d_{\mathrm{FM}}$, respectively. We also show the $V_{\mathrm{DC}}$ spectra at $f=12 \mathrm{GHz}$ for the $\operatorname{Pt}(1.5) / \mathrm{Fe}\left(d_{\mathrm{FM}}\right)$ and $\operatorname{Pt}(7.4) / \mathrm{Fe}\left(d_{\mathrm{FM}}\right)$ bilayers in Figs. 4(c) and 4(d), respectively. All the measured $V_{\mathrm{DC}}$ spectra are consistent with Eq. (1). We note that the sign of the antisymmetric component $A$ is opposite between the $\mathrm{Pt}(1.5) / \mathrm{Fe}$ and $\mathrm{Pt}(7.4) / \mathrm{Fe}$ bilayers [see Figs. 4(c) and 4(d)]. We also note that in the $\operatorname{Pt}(7.4) / \mathrm{Fe}\left(d_{\mathrm{FM}}\right)$ film, the sign of $A$ is reversed by decreasing $d_{\mathrm{FM}}$ from $3 \mathrm{~nm}$ to $2 \mathrm{~nm}$. The sign changes are induced by the competition between the Oersted field $H_{\mathrm{Oe}}$ and fieldlike effective field $H_{\mathrm{FL}}$, since $A \propto H_{\mathrm{Oe}}+H_{\mathrm{FL}}$.

To determine the dampinglike and fieldlike torque efficiencies $\xi_{\mathrm{DL}}^{E}$ and $\xi_{\mathrm{FL}}^{E}$, we plot $1 / \xi_{\mathrm{FMR}}$ at $f=9 \mathrm{GHz}$ for the $\mathrm{Pt} / \mathrm{Ni}$ bilayer and $f=16 \mathrm{GHz}$ for the $\mathrm{Pt} / \mathrm{Fe}$ bilayer as a function of $1 / d_{\mathrm{FM}}$ in Fig. 4(e). The measured values of $1 / \xi_{\mathrm{FMR}}$ is linear to $1 / d_{\mathrm{FM}}$ in all the devices, consistent with Eq. (3). The linear dependence of $1 / \xi_{\mathrm{FMR}}$ on $1 / d_{\mathrm{FM}}$ supports that the additional contributions, including the spin pumping and thermal effects, are negligible in $\xi_{\mathrm{FMR}}$. The reason for this is that in the presence of the additional contributions, $1 / \xi_{\mathrm{FMR}}$ deviates from the linear dependence on $1 / d_{\mathrm{FM}}$ [57].

Figure 4(e) shows that the sign of the intercept of the linear relation is positive in all the devices, showing that $\xi_{\mathrm{DL}}^{E}>0$ in the $\mathrm{Pt} / \mathrm{Ni}$ and $\mathrm{Pt} / \mathrm{Fe}$ bilayers because the intercept corresponds to $1 / \xi_{\text {DL }}^{E}$ [see Eq. (3)]. In contrast to the same sign of the intercept, the sign of the slope is opposite between the $\mathrm{Pt} / \mathrm{Ni}$ and $\mathrm{Pt} / \mathrm{Fe}$ bilayers. The slope of the linear relation corresponds to $\xi_{\mathrm{FL}}^{E} / \xi_{\mathrm{DL}}^{E}$, indicating that the sign of the fieldlike torque is opposite between the $\mathrm{Pt} / \mathrm{Ni}$ and $\mathrm{Pt} / \mathrm{Fe}$ bilayers: $\xi_{\mathrm{FL}}^{E}>0$ in the $\mathrm{Pt} / \mathrm{Ni}$ film and $\xi_{\mathrm{FL}}^{E}<0$ in the $\mathrm{Pt} / \mathrm{Fe}$ film. The values of $\xi_{\mathrm{DL}}^{E}$ and $\xi_{\mathrm{FL}}^{E}$, obtained by fitting the data with Eq. (3), are listed in Table I.

The result in Table I shows that the dampinglike torque efficiency $\xi_{\mathrm{DL}}^{E}$ for the $\operatorname{Pt}\left(d_{\mathrm{Pt}}\right) / \mathrm{Ni}$ and $\operatorname{Pt}\left(d_{\mathrm{Pt}}\right) / \mathrm{Fe}$ bilayers is enhanced by increasing $d_{\mathrm{Pt}}$. This result also shows that $\xi_{\mathrm{DL}}^{E}$ 
TABLE I. Parameters for the Pt/Ni and Pt/Fe bilayers, determined by the STFMR measurement: $\xi_{\mathrm{DL}}^{E}$ and $\xi_{\mathrm{FL}}^{E}$ are the dampinglike and fieldlike torque efficiencies per applied electric field $E$, respectively; $K_{\mathrm{s}}$ and $\mu_{0} M_{\mathrm{s}}$ are the interfacial perpendicular magnetic anisotropy energy density and the saturation magnetization in the FM layer, respectively, determined by fitting the FM-thickness dependence of the effective demagnetization field using Eq. (5); and $\operatorname{Re}\left[G_{\text {eff,tot }}^{\uparrow \downarrow}\right]$ is the total effective spin-mixing conductance, determined by fitting the FM-thickness dependence of the effective Gilbert magnetic damping $\alpha_{\text {eff }}$ using Eq. (6). The errors are the standard deviation determined by the fitting.

\begin{tabular}{lccccc}
\hline \hline Bilayer & $\xi_{\mathrm{DL}}^{E}\left(10^{3} \Omega^{-1} \mathrm{~cm}^{-1}\right)$ & $\xi_{\mathrm{FL}}^{E}\left(10^{3} \Omega^{-1} \mathrm{~cm}^{-1}\right)$ & $K_{\mathrm{s}}\left(\mathrm{mJ} / \mathrm{m}^{2}\right)$ & $\mu_{0} M_{\mathrm{s}}(\mathrm{T})$ & $\operatorname{Re}\left[G_{\text {eff,tot }}^{\uparrow \downarrow}\right]\left(10^{15} \Omega^{-1} \mathrm{~m}^{-2}\right)$ \\
\hline $\operatorname{Pt}(1.5) / \mathrm{Ni}$ & $1.81 \pm 0.36$ & $0.42 \pm 0.02$ & $0.05 \pm 0.02$ & $0.47 \pm 0.02$ & $1.30 \pm 0.25$ \\
$\mathrm{Pt}(7.4) / \mathrm{Ni}$ & $2.84 \pm 0.12$ & $0.53 \pm 0.05$ & $0.14 \pm 0.01$ & $0.49 \pm 0.01$ & $1.73 \pm 0.08$ \\
$\mathrm{Pt}(1.5) / \mathrm{Fe}$ & $1.75 \pm 0.15$ & $-0.77 \pm 0.03$ & $1.41 \pm 0.04$ & $2.07 \pm 0.01$ & $1.27 \pm 0.03$ \\
$\mathrm{Pt}(7.4) / \mathrm{Fe}$ & $2.99 \pm 0.11$ & $-2.18 \pm 0.11$ & $1.24 \pm 0.18$ & $1.93 \pm 0.06$ & $1.26 \pm 0.05$ \\
\hline \hline
\end{tabular}

is almost identical in the $\operatorname{Pt}\left(d_{\mathrm{Pt}}\right) / \mathrm{Ni}$ and $\operatorname{Pt}\left(d_{\mathrm{Pt}}\right) / \mathrm{Fe}$ bilayers. In contrast, the choice of the FM layer strongly affects the fieldlike torque; even the sign of $\xi_{\mathrm{FL}}^{E}$ is reversed by changing the FM layer from Ni to Fe.

\section{Magnetic anisotropy and effective spin-mixing conductance}

Before discussing the spin-torque efficiencies, we first characterize the spin-orbit coupling at the $\mathrm{Pt} / \mathrm{FM}$ interface by quantifying the magnetic anisotropy and effective spinmixing conductance, both affected by the interfacial spin-orbit coupling. The interface magnetic anisotropy can be estimated from the $1 / d_{\mathrm{FM}}$ dependence of $\mu_{0} M_{\mathrm{eff}}$ using [58]

$$
\mu_{0} M_{\mathrm{eff}}=\mu_{0} M_{\mathrm{s}}-\frac{2 K_{\mathrm{s}}}{M_{\mathrm{s}}} \frac{1}{d_{\mathrm{FM}}},
$$

where $K_{\mathrm{s}}$ and $M_{\mathrm{s}}$ are the interface perpendicular magnetic anisotropy energy density and the saturation magnetization, respectively. We determined $\mu_{0} M_{\text {eff }}$ by fitting the measured $f$ dependence of $H_{\text {FMR }}$ using the Kittel formula. In Fig. 5(a) we show the $1 / d_{\mathrm{FM}}$ dependence of $\mu_{0} M_{\text {eff }}$. By fitting the data using Eq. (5), we obtained $K_{\mathrm{s}}$ for the $\mathrm{Pt} / \mathrm{Ni}$ and $\mathrm{Pt} / \mathrm{Fe}$ films, as in Table I. Here $K_{\mathrm{s}}$ is the sum of the magnetic anisotropy energy density at the $\mathrm{Pt} / \mathrm{FM}$ and $\mathrm{FM} / \mathrm{SiO}_{2}$ interfaces: $K_{\mathrm{s}}=K_{\mathrm{s}}^{\mathrm{P} / / \mathrm{FM}}+K_{\mathrm{s}}^{\mathrm{FM} / \mathrm{SiO}_{2}}$. Since a previous study shows that $K_{\mathrm{s}}^{\mathrm{Fe} / \mathrm{SiO}_{2}}=0.6 \mathrm{~mJ} / \mathrm{m}^{2}$ [59], the result shown in Table I indicates that $K_{\mathrm{s}}^{\mathrm{Pt} / \mathrm{Fe}}$ is several times larger than $K_{\mathrm{s}}^{\mathrm{Pt} / \mathrm{Ni}}$. The larger $K_{\mathrm{s}}^{\mathrm{Pt} / \mathrm{Fe}}$ suggests that the interfacial spin-orbit coupling

(a)

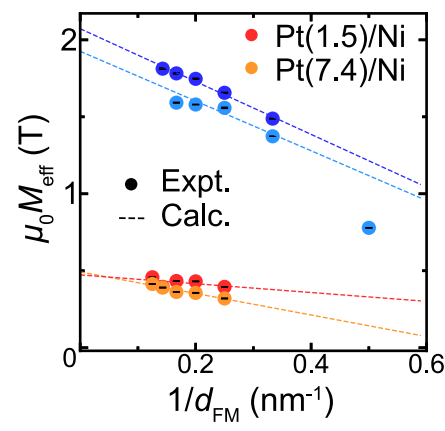

(b)

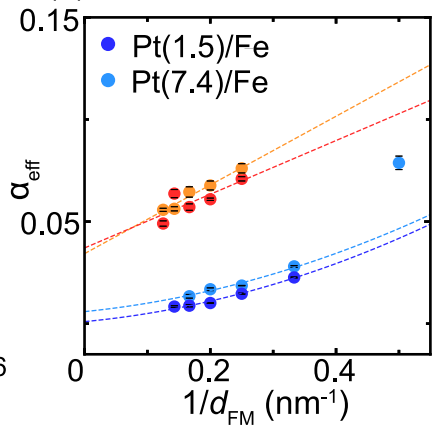

FIG. 5. (a) The $1 / d_{\mathrm{FM}}$ dependence of the effective demagnetization field $\mu_{0} M_{\text {eff }}$, where $d_{\mathrm{FM}}$ is the thickness of the FM layer. The dotted lines are the linear fitting result. (b) Effective magnetic damping $\alpha_{\text {eff }}$ as a function of $1 / d_{\mathrm{FM}}$. The dotted lines are the fitting result. at the $\mathrm{Pt} / \mathrm{Fe}$ interface is stronger than that at the $\mathrm{Pt} / \mathrm{Ni}$ interface [60].

The magnetic damping, affected by the interfacial spinorbit coupling, also supports the stronger interfacial spin-orbit coupling at the $\mathrm{Pt} / \mathrm{Fe}$ interface. In Fig. 5(b) we show the $1 / d_{\mathrm{FM}}$ dependence of the effective Gilbert damping $\alpha_{\text {eff }}$ for the $\mathrm{Pt} / \mathrm{Ni}$ and $\mathrm{Pt} / \mathrm{Fe}$ bilayers. The effective Gilbert damping $\alpha_{\text {eff }}$ was determined by fitting the measured $f$ dependence of the STFMR linewidth $W$ using $W=W_{0}+\left(2 \pi \alpha_{\text {eff }} / \gamma\right) f$, where $W_{0}$ is the inhomogeneous linewidth. The effective Gilbert magnetic damping $\alpha_{\text {eff }}$ in the presence of the spin pumping is expressed as [61]

$$
\alpha_{\text {eff }}=\alpha_{\text {int }}+\operatorname{Re}\left[G_{\text {eff,tot }}^{\uparrow \downarrow}\right] \frac{g \mu_{\mathrm{B}} h}{4 \pi e^{2} M_{\mathrm{s}}} \frac{1}{d_{\mathrm{FM}}}+\frac{\beta_{\mathrm{TMS}}}{d_{\mathrm{FM}}^{2}},
$$

where $\alpha_{\text {int }}$ is the intrinsic magnetic damping of the FM layer, $\operatorname{Re}\left[G_{\text {eff,tot }}^{\uparrow \downarrow}\right]$ is the real part of the total effective spin-mixing conductance, $g$ is the $g$ factor, $\mu_{\mathrm{B}}$ is the Bohr magnetron, and $h$ is the Planck constant. In addition, $\operatorname{Re}\left[G_{\text {eff,tot }}^{\uparrow \downarrow}\right]$ consists of two components $\operatorname{Re}\left[G_{\text {eff,tot }}^{\uparrow \downarrow}\right]=\operatorname{Re}\left[G_{\text {eff }}^{\uparrow \downarrow}\right]+G_{\text {SML }}$, where $G_{\text {eff }}^{\uparrow \downarrow}$ is the effective spin-mixing conductance, which characterizes the spin relaxation in the bulk of the Pt layer, and $G_{\mathrm{SML}}$ characterizes the strength of the spin memory loss at the Pt/FM interface. In Eq. (6) the third term is the contribution from two-magnon scattering, where the coefficient $\beta_{\mathrm{TMS}}$ depends on both $\left(K_{\mathrm{s}} / M_{\mathrm{s}}\right)^{2}$ and the density of magnetic defects at the FM surfaces [61-63]. Since the two-magnon scattering at FM/oxide interfaces is known to be relatively weak, the primary source of the two-magnon scattering in the devices used in the present study is the $\mathrm{Pt} / \mathrm{FM}$ interface. For the $\mathrm{Pt} / \mathrm{Fe}$ bilayers, $\alpha_{\text {int }}$ obtained by neglecting the $d_{\mathrm{FM}}^{-2}$ term is unphysically small or even negative, showing that the contribution from the two-magnon scattering cannot be neglected. In contrast, reasonable values of $\alpha_{\text {int }}$ were obtained without taking into account the two-magnon scattering for the $\mathrm{Pt} / \mathrm{Ni}$ bilayers. This difference is consistent with the fact that the strength of the two-magnon scattering depends on $\left(K_{\mathrm{s}} / M_{\mathrm{s}}\right)^{2}$, which is clearly different in the $\mathrm{Pt} / \mathrm{Fe}$ and $\mathrm{Pt} / \mathrm{Ni}$ films, as shown in Table I. In Table I we show $\operatorname{Re}\left[G_{\text {eff, tot }}^{\uparrow \downarrow}\right]$ for the Pt/FM bilayers, extracted by fitting the $1 / d_{\mathrm{FM}}$ dependence of $\alpha_{\text {eff }}$ in Fig. 5(b) using Eq. (6). From the fitting, we obtained $\beta_{\mathrm{TMS}}=0.1 \mathrm{~nm}^{2}$ for the $\mathrm{Pt} / \mathrm{Fe}$ bilayers, while we neglected the two-magnon scattering for the $\mathrm{Pt} / \mathrm{Ni}$ bilayers for the above reason.

Table I shows that the effective spin-mixing conductance $\operatorname{Re}\left[G_{\text {eff,tot }}^{\uparrow \downarrow}\right]$ in the Pt/Ni bilayer depends on the Pt thickness $d_{\mathrm{Pt}}$, while $\operatorname{Re}\left[G_{\text {eff,tot }}^{\uparrow \downarrow}\right]$ in the $\mathrm{Pt} / \mathrm{Fe}$ bilayer is independent 
of $d_{\mathrm{Pt}}$. Since the effective spin-mixing conductance $\operatorname{Re}\left[G_{\text {eff }}^{\uparrow \downarrow}\right]$ depends on the thickness of the Pt layer $d_{\mathrm{Pt}}$, while $G_{\mathrm{SML}}$ is independent of $d_{\mathrm{Pt}}$, this difference shows that the spin memory loss at the $\mathrm{Pt} / \mathrm{Fe}$ interface is stronger than that at the $\mathrm{Pt} / \mathrm{Ni}$ interface, supporting the stronger spin-orbit coupling at the $\mathrm{Pt} / \mathrm{Fe}$ interface. This result is consistent with the stronger two-magnon scattering at the $\mathrm{Pt} / \mathrm{Fe}$ interface because the two-magnon scattering increases with the strength of the interfacial spin-orbit coupling [64].

\section{Dampinglike torque}

Next we discuss the dampinglike torque efficiency. The measured dampinglike torque efficiency $\xi_{\mathrm{DL}}^{E}$ can be decomposed into $d_{\mathrm{Pt}}$-dependent $\xi_{\mathrm{DL}, \mathrm{dep}}^{E}\left(d_{\mathrm{Pt}}\right)$ and $d_{\mathrm{Pt}}$-independent $\xi_{\mathrm{DL}, \text { indep }}^{E}\left(d_{\mathrm{Pt}}\right)$ components: $\xi_{\mathrm{DL}}^{E}\left(d_{\mathrm{Pt}}\right)=\xi_{\mathrm{DL}, \mathrm{dep}}^{E}\left(d_{\mathrm{Pt}}\right)+\xi_{\mathrm{DL} \text {,indep }}^{E}$. One of the sources of $\xi_{\mathrm{DL} \text {,dep }}^{E}\left(d_{\mathrm{Pt}}\right)$ is the bulk spin Hall effect in the $\mathrm{Pt}$ layer. Although the dampinglike torque due to the interfacial spin current originating from interfacial spin-orbit scattering also increases with $d_{\mathrm{Pt}}[41,65]$, we first neglect the contribution from this mechanism for simplicity.

The dampinglike torque efficiency due to the bulk spin Hall effect in the simplest drift-diffusion model is expressed as $\xi_{\mathrm{DL}, \mathrm{SHE}}^{E}\left(d_{\mathrm{Pt}}\right)=\left[1-\operatorname{sech}\left(d_{\mathrm{Pt}} / \lambda_{\mathrm{S}}\right)\right] \sigma_{\mathrm{SHE}}^{\text {eff }}$, where $\sigma_{\mathrm{SHE}}^{\text {eff }}=\sigma_{\mathrm{SHE}} \varepsilon$ is the effective spin Hall conductivity of the Pt layer, where $\sigma_{\mathrm{SHE}}$ is the spin Hall conductivity and $\varepsilon$ represents the strength of the spin memory loss at the interface $(\varepsilon \leqslant 1)$. The reason for using this simple model is that this study aims to clarify the role of the bulk and interface in the generation of the spinorbit torques in the Pt/FM structures with the different FM layers, rather than precisely quantify the bulk and interfacial spin-orbit torque efficiencies.

Since the spin diffusion length $\lambda_{\mathrm{s}}$ and the resistivity $\rho_{\mathrm{Pt}}$ of Pt films are dependent on the Pt thickness $d_{\mathrm{Pt}}$, we estimate $\lambda_{\mathrm{s}}$ for each Pt/FM devices with different $d_{\mathrm{Pt}}$, assuming the ElliotYafet spin-relaxation mechanism $\lambda_{\mathrm{s}} \propto 1 / \rho_{\mathrm{Pt}}: \lambda_{\mathrm{s}}=0.98 \mathrm{~nm}$ for $d_{\mathrm{Pt}}=1.5 \mathrm{~nm}$ and $\lambda_{\mathrm{s}}=2.65 \mathrm{~nm}$ for $d_{\mathrm{Pt}}=7.4 \mathrm{~nm}$, obtained from $\rho_{\mathrm{Pt}} \lambda_{\mathrm{s}}=0.61 \times 10^{15} \Omega \mathrm{m}^{2}$ [66]. For the Pt/FM films, we also assume that the spin Hall conductivity $\sigma_{\text {SHE }}$ is independent of $d_{\mathrm{Pt}}$ because the Pt resistivity in the devices is in the moderately dirty regime [66]. Using the drift-diffusion model with the above assumptions, we obtain the change of the dampinglike torque efficiency due to the spin Hall effect as $\xi_{\mathrm{DL}, \mathrm{SHE}}^{E}\left(d_{\mathrm{Pt}}=7.4 \mathrm{~nm}\right) / \xi_{\mathrm{DL}, \mathrm{SHE}}^{E}\left(d_{\mathrm{Pt}}=1.5 \mathrm{~nm}\right)=1.42$.

From the values shown in Table I, we obtain $\xi_{\mathrm{DL}}^{E}\left(d_{\mathrm{Pt}}=\right.$ $7.4 \mathrm{~nm}) / \xi_{\mathrm{DL}}^{E}\left(d_{\mathrm{Pt}}=1.5 \mathrm{~nm}\right)=1.57 \pm 0.13$ for the $\mathrm{Pt} / \mathrm{Ni}$ bilayer and $\xi_{\mathrm{DL}}^{E}\left(d_{\mathrm{Pt}}=7.4 \mathrm{~nm}\right) / \xi_{\mathrm{DL}}^{E}\left(d_{\mathrm{Pt}}=1.5 \mathrm{~nm}\right)=1.71 \pm$ 0.05 for the $\mathrm{Pt} / \mathrm{Fe}$ bilayer. The experimental value of $\xi_{\mathrm{DL}}^{E}\left(d_{\mathrm{Pt}}=7.4 \mathrm{~nm}\right) / \xi_{\mathrm{DL}}^{E}\left(d_{\mathrm{Pt}}=1.5 \mathrm{~nm}\right)$ for the $\mathrm{Pt} / \mathrm{Ni}$ bilayer is close to the prediction of the above simple model of the spin Hall effect, suggesting that the dampinglike torque in the $\mathrm{Pt} / \mathrm{Ni}$ bilayer is dominated by the spin Hall effect. The experimental value of $\xi_{\mathrm{DL}}^{E}\left(d_{\mathrm{Pt}}=7.4 \mathrm{~nm}\right) / \xi_{\mathrm{DL}}^{E}\left(d_{\mathrm{Pt}}=1.5 \mathrm{~nm}\right)$ for the $\mathrm{Pt} / \mathrm{Fe}$ bilayer is also comparable to the prediction of the spin Hall effect. However, it is reasonable to consider that a sizable dampinglike torque is generated not only by the bulk spin Hall effect in the Pt layer but also by different mechanisms other than the spin Hall effect in the $\mathrm{Pt} / \mathrm{Fe}$ bilayer. The reason for this is that the magnitude of $\xi_{\mathrm{DL}}^{E}$ of the $\mathrm{Pt} / \mathrm{Fe}$ bilayer is comparable to that of the $\mathrm{Pt} / \mathrm{Ni}$ bilayer despite the fact that the stronger spin memory loss at the $\mathrm{Pt} / \mathrm{Fe}$ interface suppresses the dampinglike torque due to the spin Hall effect. This suggests that the dampinglike torque originating at the $\mathrm{Pt} / \mathrm{Fe}$ interface contributes to $\xi_{\mathrm{DL}}^{E}$ in the $\mathrm{Pt} / \mathrm{Fe}$ bilayer, which is consistent with the interfacial spin-orbit coupling in the $\mathrm{Pt} / \mathrm{Fe}$ bilayer being stronger than that in the $\mathrm{Pt} / \mathrm{Ni}$ bilayer.

\section{E. Fieldlike torque}

The fieldlike torque efficiency $\xi_{\mathrm{FL}}^{E}$ can also be decomposed into $d_{\mathrm{Pt}}$-dependent $\xi_{\mathrm{FL}, \text { dep }}^{E}\left(d_{\mathrm{Pt}}\right)$ and $d_{\mathrm{Pt}}$-independent $\xi_{\mathrm{FL} \text {,indep }}^{E}$ components: $\xi_{\mathrm{FL}}^{E}\left(d_{\mathrm{Pt}}\right)=\xi_{\mathrm{FL}, \text { dep }}^{E}\left(d_{\mathrm{Pt}}\right)+\xi_{\mathrm{FL} \text {,indep }}^{E}$. We note that $\xi_{\mathrm{FL}, \text { dep }}^{E}\left(d_{\mathrm{Pt}}\right)$ and $\xi_{\mathrm{FL} \text {,indep }}^{E}$ mainly originate from the bulk spin Hall effect and interfacial spin-orbit coupling, respectively; the fieldlike toque originating from the spin current due to the interfacial spin-orbit scattering, as well as the spin accumulation due to the Rashba-Edelstein effect, is insensitive to $d_{\mathrm{Pt}}$ $[41,65]$. The separation of the bulk and interface contributions from the result shown in Table I based on an established model requires us to assume various parameters, such as the real and imaginary parts of the spin-mixing conductance, as well as the spin Hall conductivity [29,67], making the analysis less reliable. Thus, we use an alternative approach to roughly estimate the bulk and interface contributions to the fieldlike torque. We assume that the fieldlike torque efficiency due to the spin Hall effect $\xi_{\mathrm{FL}, \mathrm{SHE}}^{E}\left(d_{\mathrm{Pt}}\right)$ follows the same $d_{\mathrm{Pt}}$ dependence as with the dampinglike torque efficiency $\xi_{\mathrm{DL}, \mathrm{SHE}}^{E}\left(d_{\mathrm{Pt}}\right): \xi_{\mathrm{FL}, \mathrm{SHE}}^{E}\left(d_{\mathrm{Pt}}\right)=\left[1-\operatorname{sech}\left(d_{\mathrm{Pt}} / \lambda_{\mathrm{s}}\right)\right] \xi_{\mathrm{FL}, \mathrm{SHE}}^{E, 0}$ or $\xi_{\mathrm{FL}, \mathrm{SHE}}^{E}\left(d_{\mathrm{Pt}}=7.4 \mathrm{~nm}\right) / \xi_{\mathrm{FL}, \mathrm{SHE}}^{E}\left(d_{\mathrm{Pt}}=1.5 \mathrm{~nm}\right)=1.42$.

From the values shown in Table I, we obtain $\xi_{\mathrm{FL}}^{E}\left(d_{\mathrm{Pt}}=\right.$ $7.4 \mathrm{~nm}) / \xi_{\mathrm{FL}}^{E}\left(d_{\mathrm{Pt}}=1.5 \mathrm{~nm}\right)=1.26 \pm 0.08$ for the $\mathrm{Pt} / \mathrm{Ni}$ bilayer and $\xi_{\mathrm{FL}}^{E}\left(d_{\mathrm{Pt}}=7.4 \mathrm{~nm}\right) / \xi_{\mathrm{FL}}^{E}\left(d_{\mathrm{Pt}}=1.5 \mathrm{~nm}\right)=2.83 \pm$ 0.02 for the $\mathrm{Pt} / \mathrm{Fe}$ bilayer. This result shows that $\xi_{\mathrm{FL}}^{E}\left(d_{\mathrm{Pt}}=\right.$ $7.4 \mathrm{~nm}) / \xi_{\mathrm{FL}}^{E}\left(d_{\mathrm{Pt}}=1.5 \mathrm{~nm}\right)$ for the $\mathrm{Pt} / \mathrm{Fe}$ film is clearly different from the expectation from the fieldlike torque efficiency $\xi_{\mathrm{FL}, \mathrm{SHE}}^{E}\left(d_{\mathrm{Pt}}\right)$ due to the bulk spin Hall effect. This shows that a sizable fieldlike torque is generated by the $\mathrm{Pt} / \mathrm{Fe}$ interface, which is consistent with the strong spin-orbit coupling at the $\mathrm{Pt} / \mathrm{Fe}$ interface.

In the $\mathrm{Pt} / \mathrm{Fe}$ bilayer, the clear change of $\xi_{\mathrm{FL}}^{E}$ induced by changing $d_{\mathrm{Pt}}$ indicates that a sizable fieldlike torque is also generated by the bulk spin Hall effect. To test the role of the bulk spin Hall effect in the fieldlike torque efficiency $\xi_{\mathrm{FL}}^{E}$, we define the change of the fieldlike torque efficiency $\Delta \xi_{\mathrm{FL}}^{E}$ as $\Delta \xi_{\mathrm{FL}}^{E}=\xi_{\mathrm{FL}}^{E}(7.4 \mathrm{~nm})-\xi_{\mathrm{FL}}^{E}(1.5 \mathrm{~nm})=$ $\xi_{\mathrm{FL}, \mathrm{dep}}^{E}(7.4 \mathrm{~nm})-\xi_{\mathrm{FL}, \mathrm{dep}}^{E}(1.5 \mathrm{~nm})$. From the result shown in Table I, we obtain $\Delta \xi_{\mathrm{FL}}^{E}>0$ for the $\mathrm{Pt} / \mathrm{Ni}$ bilayer, while $\Delta \xi_{\mathrm{FL}}^{E}<0$ for the $\mathrm{Pt} / \mathrm{Fe}$ bilayer. Since the nonzero value of $\Delta \xi_{\mathrm{FL}}^{E}$ arises from the $d_{\mathrm{Pt}}$-dependent component of the fieldlike torque, the opposite sign of $\Delta \xi_{\mathrm{FL}}^{E}$ shows that the sign of the fieldlike torque due to the bulk spin Hall effect in the Pt layer is opposite in the $\mathrm{Pt} / \mathrm{Ni}$ and $\mathrm{Pt} / \mathrm{Fe}$ bilayers. We also note that the fieldlike torque efficiency $\xi_{\mathrm{FL}}^{E}$ in Table I shows that the magnitude of the fieldlike torque in the $\mathrm{Pt} / \mathrm{Fe}$ bilayer is clearly larger than that in the $\mathrm{Pt} / \mathrm{Ni}$ bilayer. Since the above result is a rough estimation, we only focus on the sign and relative magnitude of the fieldlike torque below.

The interfacial spin-orbit coupling plays an important role in the generation of the fieldlike torque due to the bulk spin 
Hall effect, as well as that arising from the interface. In the model of the spin-orbit torques due to the bulk spin Hall effect, the real part of the spin-mixing conductance $\operatorname{Re}\left[G_{\mathrm{Pt} / \mathrm{FM}}^{\uparrow \downarrow}\right]$ contributes to the dampinglike torque, whereas the imaginary part $\operatorname{Im}\left[G_{\mathrm{Pt} / \mathrm{FM}}^{\uparrow \downarrow}\right]$ contributes to the fieldlike torque [29]. The reason for this is that the fieldlike torque due to the spin Hall effect arises from the reflection of the spin current at the interface, while the dampinglike torque arises from the injection of the spin current. Although $\operatorname{Im}\left[G_{\mathrm{Pt} / \mathrm{FM}}^{\uparrow \downarrow}\right]$ has been believed to be negligible compared to $\operatorname{Re}\left[G_{\mathrm{Pt} / \mathrm{FM}}^{\uparrow \downarrow}\right]$ in metallic films, recent studies have shown non-negligible $\operatorname{Im}\left[G_{\mathrm{Pt} / \mathrm{FM}}^{\uparrow \downarrow}\right]$ in such systems $[43,68-70]$. Since sizable $\operatorname{Im}\left[G_{\mathrm{Pt} / \mathrm{FM}}^{\uparrow \downarrow}\right]$ appears when a spin current reflected at the interface experiences a large angle rotation of its spin direction due to interfacial spin-orbit coupling [68], the stronger fieldlike torque in the $\mathrm{Pt} / \mathrm{Fe}$ film, compared with that in the $\mathrm{Pt} / \mathrm{Ni}$ film, is consistent with the stronger spin-orbit coupling at the $\mathrm{Pt} / \mathrm{Fe}$ interface.

The opposite sign of the fieldlike torque in the $\mathrm{Pt} / \mathrm{Ni}$ and $\mathrm{Pt} / \mathrm{Fe}$ bilayers indicates that the sign of $\operatorname{Im}\left[G_{\mathrm{Pt} / \mathrm{FM}}^{\uparrow \downarrow}\right]$ is opposite in these devices. Here the sign of the imaginary part of the spin-mixing conductance can be different depending on the choice of the FM layer. For a model where all of the Fermi surfaces are spherical and the same size, the real and imaginary parts of the spin-mixing conductance due to spindependent reflection arising from a spin-dependent potential at the interface are expressed as [29,67]

$$
\begin{aligned}
\operatorname{Re}\left[G_{\mathrm{Pt} / \mathrm{FM}}^{\uparrow \downarrow}\right] & =\frac{e^{2} k_{\mathrm{F}}^{2}}{2 \pi h}\left\{\frac{1}{2}+\frac{u^{\uparrow} u^{\downarrow}}{2\left(u^{\uparrow}+u^{\downarrow}\right)}\left[u^{\downarrow} \ln \left(\frac{u^{\downarrow^{2}}}{1+u^{\downarrow^{2}}}\right)+u^{\uparrow} \ln \left(\frac{u^{\uparrow^{2}}}{1+u^{\uparrow^{2}}}\right)\right]\right\}, \\
\operatorname{Im}\left[G_{\mathrm{Pt} / \mathrm{FM}}^{\uparrow \downarrow}\right] & =\frac{e^{2} k_{\mathrm{F}}^{2}}{2 \pi h}\left(\frac{u^{\uparrow} u^{\downarrow}}{2\left(u^{\uparrow}+u^{\downarrow}\right)}\left(u^{\downarrow}\left[\pi-2 \tan ^{-1} u^{\downarrow}\right]-u^{\uparrow}\left[\pi-2 \tan ^{-1} u^{\uparrow}\right]\right)\right) .
\end{aligned}
$$

Here $u^{\uparrow}$ and $u^{\downarrow}$ represent the strength of the spin-dependent potential at the interface, where $\uparrow$ and $\downarrow$ refer to majority and minority electrons, respectively. Equations (7) and (8) show that the sign of $\operatorname{Re}\left[G_{\mathrm{Pt} / \mathrm{FM}}^{\uparrow \downarrow}\right]$ is always positive. In contrast, the sign of $\operatorname{Im}\left[G_{\mathrm{Pt} / \mathrm{FM}}^{\uparrow \downarrow}\right]$ depends on the relative strength between the spin-dependent potentials $u^{\uparrow}$ and $u^{\downarrow}$. This indicates that the the sign of the fieldlike torque due to the spin Hall effect depends on the electronic structure of the FM layer.

\section{CONCLUSION}

In summary, we have investigated the dampinglike and fieldlike torque efficiencies in the $\mathrm{Pt} / \mathrm{Ni}$ and $\mathrm{Pt} / \mathrm{Fe}$ bilayers with different Pt-layer thicknesses to reveal the origin of the spin-orbit torques in the Pt-based structures. We found that the dampinglike torque efficiency $\xi_{\mathrm{DL}}^{E}$ is enhanced by increasing $d_{\mathrm{Pt}}$ in both $\mathrm{Pt} / \mathrm{Ni}$ and $\mathrm{Pt} / \mathrm{Fe}$ bilayers. The result also shows that the magnitude of $\xi_{\mathrm{DL}}^{E}$ is almost identical in the $\mathrm{Pt} / \mathrm{Ni}$ and $\mathrm{Pt} / \mathrm{Fe}$ bilayers despite the stronger spin memory loss at the $\mathrm{Pt} / \mathrm{Fe}$ interface. These results suggest that although the $d_{\mathrm{Pt}}$ dependence of $\xi_{\mathrm{DL}}^{E}$ is consistent with the dampinglike torque due to the bulk spin Hall effect in the $\mathrm{Pt}$ layer, the $\mathrm{Pt} / \mathrm{Fe}$ interface also contributes to the dampinglike torque in the $\mathrm{Pt} / \mathrm{Fe}$ bilayer. The non-negligible contribution from the interface to the dampinglike torque is consistent with the spin-orbit coupling at the $\mathrm{Pt} / \mathrm{Fe}$ interface being stronger than that at the $\mathrm{Pt} / \mathrm{Ni}$ interface. Although a part of the dampinglike torque in the $\mathrm{Pt} / \mathrm{Fe}$ bilayer originates from the interface, the origin is unclear from the result; the intrinsic spin-orbit torque and/or the interfacial spin-orbit scattering may play an important role in the interface generation of the dampinglike torque.

In contrast to the dampinglike torque, whose magnitude and sign are almost identical in the $\mathrm{Pt} / \mathrm{Ni}$ and $\mathrm{Pt} / \mathrm{Fe}$ bilayers, the fieldlike torque strongly depends on the choice of the FM layer. In particular, the sign of the $d_{\mathrm{Pt}}$-dependent component of the fieldlike torque efficiency $\xi_{\mathrm{FL}}^{E}$ is opposite between the
$\mathrm{Pt} / \mathrm{Ni}$ and $\mathrm{Pt} / \mathrm{Fe}$ bilayers. This indicates that the direction of the fieldlike torque due to the bulk spin Hall effect in the $\mathrm{Pt}$ layer is opposite between the $\mathrm{Pt} / \mathrm{Ni}$ and $\mathrm{Pt} / \mathrm{Fe}$ bilayers, which is attributed to the opposite sign of the imaginary part of the spin-mixing conductance. The magnitude, as well as the sign, of the fieldlike torque is also clearly different in the $\mathrm{Pt} / \mathrm{Fe}$ and $\mathrm{Pt} / \mathrm{Ni}$ bilayers. The stronger fieldlike torque in the $\mathrm{Pt} / \mathrm{Fe}$ bilayer indicates that the imaginary part of the spin-mixing conductance in the $\mathrm{Pt} / \mathrm{Fe}$ bilayer is larger than that in the $\mathrm{Pt} / \mathrm{Ni}$ bilayer, which is also consistent with the stronger interfacial spin-orbit coupling in the $\mathrm{Pt} / \mathrm{Fe}$ bilayer.

Previous studies have demonstrated that the interfacial spin-orbit coupling is also strong in $\mathrm{Pt} / \mathrm{Co}$ bilayers. However, the role of the bulk and interfacial spin-orbit coupling in the generation of the spin-orbit torques is clearly different between the $\mathrm{Pt} / \mathrm{Co}$ and $\mathrm{Pt} / \mathrm{Fe}$ bilayers. The dominant source of the dampinglike torque in the $\mathrm{Pt} / \mathrm{Co}$ bilayer is the bulk spin Hall effect in the Pt layer, which is the same as that in the $\mathrm{Pt} / \mathrm{Ni}$ and $\mathrm{Pt} / \mathrm{Fe}$ bilayers $[64,71,72]$. In contrast, the fieldlike torque in the $\mathrm{Pt} / \mathrm{Co}$ bilayer is mainly generated by the $\mathrm{Pt} / \mathrm{Co}$ interface; the contribution from the bulk spin Hall effect to the fieldlike torque is negligible in the $\mathrm{Pt} / \mathrm{Co}$ bilayer. This is different from the fieldlike torque in the $\mathrm{Pt} / \mathrm{Fe}$ bilayer, where the bulk spin Hall effect plays an important role, even though both films show the strong interfacial spin-orbit coupling. The different mechanisms responsible for the spin-orbit torques in the Pt/FM bilayers show that the electronic structure of the FM layer, as well as the interfacial spin-orbit coupling, plays an important role in the generation of the spin-orbit torques.

\section{ACKNOWLEDGMENTS}

This work was supported by JSPS KAKENHI Grants No. 19H00864 and No. 19K22131, Canon Foundation, Asahi Glass Foundation, Kao Foundation for Arts and Sciences, JGC-S Scholarship Foundation, and Spintronics Research Network of Japan. 
[1] A. Manchon, J. Železný, I. M. Miron, T. Jungwirth, J. Sinova, A. Thiaville, K. Garello, and P. Gambardella, Current-induced spin-orbit torques in ferromagnetic and antiferromagnetic systems, Rev. Mod. Phys. 91, 035004 (2019).

[2] I. M. Miron, K. Garello, G. Gaudin, P.-J. Zermatten, M. V. Costache, S. Auffret, S. Bandiera, B. Rodmacq, A. Schuhl, and P. Gambardella, Perpendicular switching of a single ferromagnetic layer induced by in-plane current injection, Nature (London) 476, 189 (2011).

[3] L. Liu, C.-F. Pai, Y. Li, H. Tseng, D. Ralph, and R. Buhrman, Spin-torque switching with the giant spin Hall effect of tantalum, Science 336, 555 (2012).

[4] G. Yu, P. Upadhyaya, Y. Fan, J. G. Alzate, W. Jiang, K. L. Wong, S. Takei, S. A. Bender, L.-T. Chang, Y. Jiang, M. Lang, J. Tang, Y. Wang, Y. Tserkovnyak, P. K. Amiri, and K. L. Wang, Switching of perpendicular magnetization by spin-orbit torques in the absence of external magnetic fields, Nat. Nanotechnol. 9, 548 (2014).

[5] H. Kurebayashi, J. Sinova, D. Fang, A. C. Irvine, T. D. Skinner, J. Wunderlich, V. Novák, R. P. Campion, B. L. Gallagher, E. K. Vehstedt, L. P. Zârbo, K. Výborný, A. J. Ferguson, and T. Jungwirth, An antidamping spin-orbit torque originating from the Berry curvature, Nat. Nanotechnol. 9, 211 (2014).

[6] S. Fukami, C. Zhang, S. DuttaGupta, A. Kurenkov, and H. Ohno, Magnetization switching by spin-orbit torque in an antiferromagnet-ferromagnet bilayer system, Nat. Mater. 15, 535 (2016)

[7] C. O. Avci, A. Quindeau, C.-F. Pai, M. Mann, L. Caretta, A. S. Tang, M. C. Onbasli, C. A. Ross, and G. S. Beach, Currentinduced switching in a magnetic insulator, Nat. Mater. 16, 309 (2017).

[8] Y. Kageyama, Y. Tazaki, H. An, T. Harumoto, T. Gao, J. Shi, and K. Ando, Spin-orbit torque manipulated by fine-tuning of oxygen-induced orbital hybridization, Sci. Adv. 5, eaax4278 (2019).

[9] H. An, Y. Kageyama, Y. Kanno, N. Enishi, and K. Ando, Spintorque generator engineered by natural oxidation of $\mathrm{Cu}$, Nat. Commun. 7, 13069 (2016).

[10] H. Nakayama, Y. Kanno, H. An, T. Tashiro, S. Haku, A. Nomura, and K. Ando, Rashba-Edelstein Magnetoresistance in Metallic Heterostructures, Phys. Rev. Lett. 117, 116602 (2016).

[11] H. An, S. Haku, Y. Kanno, H. Nakayama, H. Maki, J. Shi, and K. Ando, Manipulation of Spin-Torque Generation using Ultrathin Au, Phys. Rev. Appl. 9, 064016 (2018).

[12] X. Qiu, K. Narayanapillai, Y. Wu, P. Deorani, D.-H. Yang, W.-S. Noh, J.-H. Park, K.-J. Lee, H.-W. Lee, and H. Yang, Spin-orbittorque engineering via oxygen manipulation, Nat. Nanotechnol. 10, 333 (2015).

[13] Y. Fan, X. Kou, P. Upadhyaya, Q. Shao, L. Pan, M. Lang, X. Che, J. Tang, M. Montazeri, K. Murata, L.-T. Chang, M. Akyol, G. Yu, T. Nie, K. L. Wong, J. Liu, Y. Wang, Y. Tserkovnyak, and K. L. Wang, Electric-field control of spin-orbit torque in a magnetically doped topological insulator, Nat. Nanotechnol. 11, 352 (2016).

[14] C. Safranski, E. A. Montoya, and I. N. Krivorotov, Spin-orbit torque driven by a planar Hall current, Nat. Nanotechnol. 14, 27 (2019).
[15] K. Kondou, R. Yoshimi, A. Tsukazaki, Y. Fukuma, J. Matsuno, K. Takahashi, M. Kawasaki, Y. Tokura, and Y. Otani, Fermilevel-dependent charge-to-spin current conversion by Dirac surface states of topological insulators, Nat. Phys. 12, 1027 (2016).

[16] J. Kim, J. Sinha, M. Hayashi, M. Yamanouchi, S. Fukami, T. Suzuki, S. Mitani, and H. Ohno, Layer thickness dependence of the current-induced effective field vector in $\mathrm{Ta}|\mathrm{CoFeB}| \mathrm{MgO}$, Nat. Mater. 12, 240 (2013).

[17] S. Manipatruni, D. E. Nikonov, C.-C. Lin, T. A. Gosavi, H Liu, B. Prasad, Y.-L. Huang, E. Bonturim, R. Ramesh, and I. A. Young, Scalable energy-efficient magnetoelectric spinorbit logic, Nature (London) 565, 35 (2019).

[18] M. Dyakonov and V. Perel, Current-induced spin orientation of electrons in semiconductors, Phys. Lett. A 35, 459 (1971).

[19] J. E. Hirsch, Spin Hall Effect, Phys. Rev. Lett. 83, 1834 (1999).

[20] J. Sinova, D. Culcer, Q. Niu, N. A. Sinitsyn, T. Jungwirth, and A. H. MacDonald, Universal Intrinsic Spin Hall Effect, Phys. Rev. Lett. 92, 126603 (2004).

[21] S. Murakami, N. Nagaosa, and S. C. Zhang, Dissipationless quantum spin current at room temperature, Science 301, 1348 (2003).

[22] Y. K. Kato, R. C. Myers, A. C. Gossard, and D. D. Awschalom, Observation of the spin Hall effect in semiconductors, Science 306, 1910 (2004).

[23] T. Kimura, Y. Otani, T. Sato, S. Takahashi, and S. Maekawa, Room-Temperature Reversible Spin Hall Effect, Phys. Rev. Lett. 98, 156601 (2007).

[24] J. Wunderlich, B. Kaestner, J. Sinova, and T. Jungwirth, Experimental Observation of the Spin-Hall Effect in a TwoDimensional Spin-Orbit Coupled Semiconductor System, Phys. Rev. Lett. 94, 047204 (2005).

[25] K. Ando, S. Takahashi, K. Harii, K. Sasage, J. Ieda, S. Maekawa, and E. Saitoh, Electric Manipulation of Spin Relaxation Using the Spin Hall Effect, Phys. Rev. Lett. 101, 036601 (2008).

[26] J. Sinova, S. O. Valenzuela, J. Wunderlich, C. H. Back, and T. Jungwirth, Spin Hall effects, Rev. Mod. Phys. 87, 1213 (2015).

[27] Y. Niimi and Y. Otani, Reciprocal spin Hall effects in conductors with strong spin-orbit coupling: A review, Rep. Prog. Phys. 78, 124501 (2015).

[28] A. Hoffmann, Spin Hall effects in metals, IEEE Trans. Magn. 49, 5172 (2013).

[29] P. M. Haney, H.-W. Lee, K.-J. Lee, A. Manchon, and M. D. Stiles, Current induced torques and interfacial spin-orbit coupling: Semiclassical modeling, Phys. Rev. B 87, 174411 (2013).

[30] E. I. Rashba, Properties of semiconductors with an extremum loop. 1. Cyclotron and combinational resonance in a magnetic field perpendicular to the plane of the loop, Sov. Phys. Solid State 2, 1109 (1960).

[31] A. Manchon, H. C. Koo, J. Nitta, S. Frolov, and R. Duine, New perspectives for Rashba spin-orbit coupling, Nat. Mater. 14, 871 (2015).

[32] V. M. Edelstein, Spin polarization of conduction electrons induced by electric current in two-dimensional asymmetric electron systems, Solid State Commun. 73, 233 (1990).

[33] V. V. Bel'kov and S. D. Ganichev, Magneto-gyrotropic effects in semiconductor quantum wells, Semicond. Sci. Technol. 23, 114003 (2008). 
[34] A. Manchon and S. Zhang, Theory of nonequilibrium intrinsic spin torque in a single nanomagnet, Phys. Rev. B 78, 212405 (2008).

[35] I. M. Miron, G. Gaudin, S. Auffret, B. Rodmacq, A. Schuhl, S. Pizzini, J. Vogel, and P. Gambardella, Current-driven spin torque induced by the Rashba effect in a ferromagnetic metal layer, Nat. Mater. 9, 230 (2010).

[36] H. Li, H. Gao, L. P. Zârbo, K. Výborný, X. Wang, I. Garate, F. Doğan, A. Čejchan, J. Sinova, T. Jungwirth, and A. Manchon, Intraband and interband spin-orbit torques in noncentrosymmetric ferromagnets, Phys. Rev. B 91, 134402 (2015).

[37] A. Qaiumzadeh, R. A. Duine, and M. Titov, Spin-orbit torques in two-dimensional Rashba ferromagnets, Phys. Rev. B 92, 014402 (2015).

[38] F. Freimuth, S. Blügel, and Y. Mokrousov, Spin-orbit torques in $\mathrm{Co} / \mathrm{Pt}(111)$ and $\mathrm{Mn} / \mathrm{W}(001)$ magnetic bilayers from first principles, Phys. Rev. B 90, 174423 (2014).

[39] T. Gao, A. Qaiumzadeh, H. An, A. Musha, Y. Kageyama, J. Shi, and K. Ando, Intrinsic Spin-Orbit Torque Arising from the Berry Curvature in a Metallic-Magnet/Cu-Oxide Interface, Phys. Rev. Lett. 121, 017202 (2018).

[40] A. Bose, H. Singh, V. K. Kushwaha, S. Bhuktare, S. Dutta, and A. A. Tulapurkar, Sign Reversal of Fieldlike Spin-Orbit Torque in an Ultrathin Cr/Ni Bilayer, Phys. Rev. Appl. 9, 014022 (2018).

[41] V. P. Amin and M. D. Stiles, Spin transport at interfaces with spin-orbit coupling: Phenomenology, Phys. Rev. B 94, 104420 (2016).

[42] L. Zhu, L. Zhu, M. Sui, D. C. Ralph, and R. A. Buhrman, Variation of the giant intrinsic spin Hall conductivity of Pt with carrier lifetime, Sci. Adv. 5, eaav8025 (2019).

[43] T. Nan, S. Emori, C. T. Boone, X. Wang, T. M. Oxholm, J. G. Jones, B. M. Howe, G. J. Brown, and N. X. Sun, Comparison of spin-orbit torques and spin pumping across $\mathrm{NiFe} / \mathrm{Pt}$ and $\mathrm{NiFe} / \mathrm{Cu} / \mathrm{Pt}$ interfaces, Phys. Rev. B 91, 214416 (2015).

[44] H. An, T. Ohno, Y. Kanno, Y. Kageyama, Y. Monnai, H. Maki, J. Shi, and K. Ando, Current-induced magnetization switching using an electrically insulating spin-torque generator, Sci. Adv. 4, eaar2250 (2018).

[45] R. Ramaswamy, Y. Wang, M. Elyasi, M. Motapothula, T. Venkatesan, X. Qiu, and H. Yang, Extrinsic Spin Hall Effect in $\mathrm{Cu}_{1-x} \mathrm{Pt}_{x}$, Phys. Rev. Appl. 8, 024034 (2017).

[46] H.-Y. Lee, S. Kim, J.-Y. Park, Y.-W. Oh, S.-Y. Park, W. Ham, Y. Kotani, T. Nakamura, M. Suzuki, T. Ono, K.-J. Lee, and B.-G. Park, Enhanced spin orbit torque via interface engineering in $\mathrm{Pt} / \mathrm{CoFeB} / \mathrm{MgO}$ heterostructures, APL Mater. 7, 031110 (2019).

[47] H. An, Y. Kanno, A. Asami, and K. Ando, Giant spin-torque generation by heavily oxidized Pt, Phys. Rev. B 98, 014401 (2018).

[48] A. J. Berger, E. R. J. Edwards, H. T. Nembach, O. Karis, M. Weiler, and T. J. Silva, Determination of the spin Hall effect and the spin diffusion length of Pt from self-consistent fitting of damping enhancement and inverse spin-orbit torque measurements, Phys. Rev. B 98, 024402 (2018).

[49] X. Fan, J. Wu, Y. Chen, M. J. Jerry, H. Zhang, and J. Q. Xiao, Observation of the nonlocal spin-orbital effective field, Nat. Commun. 4, 1799 (2013).

[50] Y. Li, H. Saglam, Z. Zhang, R. Bidthanapally, Y. Xiong, J. E. Pearson, V. Novosad, H. Qu, G. Srinivasan, A. Hoffmann, and W. Zhang, Simultaneous Optical and Electrical Spin-Torque
Magnetometry with Phase-Sensitive Detection of Spin Precession, Phys. Rev. Appl. 11, 034047 (2019).

[51] L. Liu, T. Moriyama, D. C. Ralph, and R. A. Buhrman, SpinTorque Ferromagnetic Resonance Induced by the Spin Hall Effect, Phys. Rev. Lett. 106, 036601 (2011).

[52] C.-F. Pai, Y. Ou, L. H. Vilela-Leão, D. C. Ralph, and R. A. Buhrman, Dependence of the efficiency of spin Hall torque on the transparency of Pt/ferromagnetic layer interfaces, Phys. Rev. B 92, 064426 (2015).

[53] M.-H. Nguyen, D. C. Ralph, and R. A. Buhrman, Spin Torque Study of the Spin Hall Conductivity and Spin Diffusion Length in Platinum Thin Films with Varying Resistivity, Phys. Rev. Lett. 116, 126601 (2016).

[54] D. Fang, H. Kurebayashi, J. Wunderlich, K. Výborný, L. P. Zârbo, R. Campion, A. Casiraghi, B. Gallagher, T. Jungwirth, and A. Ferguson, Spin-orbit-driven ferromagnetic resonance, Nat. Nanotechnol. 6, 413 (2011).

[55] K. Harii, T. An, Y. Kajiwara, K. Ando, H. Nakayama, T. Yoshino, and E. Saitoh, Frequency dependence of spin pumping in $\mathrm{Pt} / \mathrm{Y}_{3} \mathrm{Fe}_{5} \mathrm{O}_{12}$ film, J. Appl. Phys. 109, 116105 (2011).

[56] D. Lee, W. Jeong, D. Yun, S.-Y. Park, B.-K. Ju, K.-J. Lee, H. C. Koo, B.-C. Min, and O. Lee, Influences of interfacial oxidization on surface magnetic energy, magnetic damping and spin-orbit-torques in $\mathrm{Pt} /$ ferromagnet/capping structures, arXiv:1901.05777.

[57] S. Karimeddiny, J. A. Mittelstaedt, R. A. Buhrman, and D. C. Ralph, Transverse and Longitudinal Spin-Torque Ferromagnetic Resonance for Improved Measurement of Spin-Orbit Torque, Phys. Rev. Appl. 14, 024024 (2020).

[58] V. Tshitoyan, C. Ciccarelli, A. P. Mihai, M. Ali, A. C. Irvine, T. A. Moore, T. Jungwirth, and A. J. Ferguson, Electrical manipulation of ferromagnetic NiFe by antiferromagnetic IrMn, Phys. Rev. B 92, 214406 (2015).

[59] M. Senda and Y. Nagai, Perpendicular magnetic anisotropy of $\mathrm{Fe} / \mathrm{SiO}_{2}$ multilayer film, J. Appl. Phys. 68, 3508 (1990).

[60] L. Zhu, D. C. Ralph, and R. A. Buhrman, Spin-Orbit Torques in Heavy-Metal-Ferromagnet Bilayers with Varying Strengths of Interfacial Spin-Orbit Coupling, Phys. Rev. Lett. 122, 077201 (2019).

[61] L. Zhu, D. C. Ralph, and R. A. Buhrman, Effective Spin-Mixing Conductance of Heavy-Metal-Ferromagnet Interfaces, Phys. Rev. Lett. 123, 057203 (2019).

[62] R. Arias and D. L. Mills, Extrinsic contributions to the ferromagnetic resonance response of ultrathin films, Phys. Rev. B 60, 7395 (1999).

[63] A. Azevedo, A. B. Oliveira, F. M. de Aguiar, and S. M. Rezende, Extrinsic contributions to spin-wave damping and renormalization in thin $\mathrm{Ni}_{50} \mathrm{Fe}_{50}$ films, Phys. Rev. B 62, 5331 (2000).

[64] L. Zhu, L. Zhu, D. C. Ralph, and R. A. Buhrman, Origin of Strong Two-Magnon Scattering in HeavyMetal/Ferromagnet/Oxide Heterostructures, Phys. Rev. Appl. 13, 034038 (2020).

[65] A. Ghosh, K. Garello, C. O. Avci, M. Gabureac, and P. Gambardella, Interface-Enhanced Spin-Orbit Torques and Current-Induced Magnetization Switching of $\mathrm{Pd} / \mathrm{Co} / \mathrm{AlO}_{x}$ Layers, Phys. Rev. Appl. 7, 014004 (2017).

[66] E. Sagasta, Y. Omori, M. Isasa, M. Gradhand, L. E. Hueso, Y. Niimi, Y. C. Otani, and F. Casanova, Tuning the spin Hall effect 
of Pt from the moderately dirty to the superclean regime, Phys. Rev. B 94, 060412(R) (2016).

[67] G. Allen, S. Manipatruni, D. E. Nikonov, M. Doczy, and I. A. Young, Experimental demonstration of the coexistence of spin Hall and Rashba effects in $\beta$-tantalum/ferromagnet bilayers, Phys. Rev. B 91, 144412 (2015).

[68] J. Kim, J. Sinha, S. Mitani, M. Hayashi, S. Takahashi, S. Maekawa, M. Yamanouchi, and H. Ohno, Anomalous temperature dependence of current-induced torques in $\mathrm{CoFeB} / \mathrm{MgO}$ heterostructures with Ta-based underlayers, Phys. Rev. B 89, 174424 (2014).

[69] C.-F. Pai, M.-H. Nguyen, C. Belvin, L. H. Vilela-Leao, D. C. Ralph, and R. A. Buhrman, Enhancement of perpendicular magnetic anisotropy and transmission of spinhall-effect-induced spin currents by a $\mathrm{Hf}$ spacer layer in
W/Hf/CoFeB/MgO layer structures, Appl. Phys. Lett. 104, 082407 (2014).

[70] J. Dubowik, P. Graczyk, A. Krysztofik, H. Głowiński, E. Coy, K. Załęski, and I. Gościańska, Non-Negligible Imaginary Part of the Spin-Mixing Conductance and its Impact on Magnetization Dynamics in Heavy-Metal-Ferromagnet Bilayers, Phys. Rev. Appl. 13, 054011 (2020).

[71] Y. Du, H. Gamou, S. Takahashi, S. Karube, M. Kohda, and J. Nitta, Disentanglement of Spin-Orbit Torques in Pt/Co Bilayers with the Presence of Spin Hall Effect and Rashba-Edelstein Effect, Phys. Rev. Appl. 13, 054014 (2020).

[72] F. Mahfouzi, R. Mishra, P.-H. Chang, H. Yang, and N. Kioussis, Microscopic origin of spin-orbit torque in ferromagnetic heterostructures: A first-principles approach, Phys. Rev. B 101, 060405(R) (2020). 